\title{
Establishment of "A-PPNS", A Navigation System for Regenerating the Software Development Business
}

\author{
Hirotake Sakai ${ }^{\dagger}$ \\ School of Science and Engineering \\ Aoyama Gakuin University, Sagamihara, Kanagawa, 229-8558, JAPAN \\ Tel: +81-42-759-6423, E-mail: sakai.hirotake@gmail.com \\ Yoshihiro Waji \\ School of Science and Engineering \\ Aoyama Gakuin University, Sagamihara, Kanagawa, 229-8558, JAPAN \\ Tel: +81-42-759-6423 \\ Mari Nakamura \\ School of Science and Engineering \\ Aoyama Gakuin University, Sagamihara, Kanagawa, 229-8558, JAPAN \\ Tel: +81-42-759-6423 \\ Kakuro Amasaka \\ School of Science and Engineering \\ Aoyama Gakuin University, Sagamihara, Kanagawa, 229-8558, JAPAN \\ Tel: +81-42-759-6313, E-mail: kakuro_amasaka@ise.aoyama.ac.jp
}

Received, January 22, 2011; Revised, February 18, 2011; Accepted, February 21, 2011

\begin{abstract}
Currently, knowledge within the field of software development is largely implicit and is not formally disseminated and shared. This means that there is little improvement and regeneration of processes, and knowledge gained from previous projects is not necessarily applied to new ones. In order to turn this situation around it is necessary to take an organized approach to sharing job-related information. For this study, the authors constructed "Amalab-Project Planning Navigation System, or A-PPNS", a system for organizing accumulated knowledge related to the field of software development. More specifically, A-PPNS is a business process monitoring system and consists of the following four elements: (i) Optimized estimate support subsystem, (ii) Schedule monitoring system, (iii) QCD optimization diagnostic system, and (iv) Strategic technology accumulation system. The effectiveness of this system has been demonstrated and verified at Company A, a system integration company.
\end{abstract}

Keywords: Project Management, Software Development, Software Estimation, Strategic Technology Accumulation, "Amalab-Project Planning Navigation System, A-PPNS"

\section{INTRODUCTION}

In this article, the authors have established a systematic approach for the visualization of software development work and built the Amalab-Project Planning Navigation System A-PPNS, which adopts a diagnostic functional approach that enables responses to be made.

At Level 1 of the system, a work flow is used to make the work processes visible, and at Level 2, the "Business Process Monitoring System, A-PPNS-BPMS," manages the progress of the work and of costs.

A-PPNS is a navigation system regeneration of the software development business that takes advantage of the past accumulation of technology to enable responses to be made. Verification was made using a certain systems integrator business and the given outcomes were

$\uparrow$ : Corresponding Author 
obtained.

\section{BACKGROUND}

Despite the evolution of technology, the work of software development relies in many ways on the quality of the engineers who do the work. Information technology has become indispensable to society, but many problems exist (Otsuka, 2008). Effective management of complex software projects depends on the ability to solve complex and subtle optimization problems (Changa, 2008).

The authors have stressed the importance of visualizing work processes. This is because while work processes are becoming more sophisticated and complex, they are increasingly being developed implicitly and solely through the innate abilities of those in charge of performing them.

People are now clamoring for much-needed work innovations and reforms, and the essential first step is clearly visualizing one's own work processes (Kato, 2007; Nakamura et al., 2008).

\subsection{Current State of Software Development}

One aspect of the current state of the systems integrator, or SI industry that cannot be ignored is the problem of how to pass the work knowhow and the knowledge and technologies of information systems down to others who come after. It is well known that inaccurate or inappropriate estimations lie behind many of the problems in software development, such as delays in delivery or quality defects (Galorath and Evans, 2008).

Afsharian and Giacomobono (2008) claimed that in today's highly competitive world, accurate software estimation can make the difference between successful projects and dismal failures. Proper project planning and control is not possible without a sound and reliable estimate. And a framework developed by Ericsson R\&D (Research and Development), for project time and cost estimation for software development projects in the telecommunications domain was proposed. Mittas and Angelis (2010) proposed a formal framework covering different aspects of the estimation process such as the calibration of the prediction methodology, the identification of factors that affect the error, the investigation of errors on certain ranges of the actual cost and the examination of the distribution of the cost for certain errors. Pendharkar and Rodger (2009) studied the relationship between team size and software development cost. See also (Heričko and Živkoviča, 2008; Heričko et al., 2008).

Information systems have progressed and expanded with such diversity that it impacts on company management and on society. The required technological fields have become increasingly broad and segmentalized, requiring specialized skills. This has led to an increase in the number of engineers involved in this field and a high cost structure has become a major problem for the industry. As solutions to these problems, in addition to the multi-skilling approach of human resources development programs employed in the manufacturing industry, proposals must be developed for response directions that will achieve productivity improvements and ensure quality through the re-use of work knowledge and software development outcomes (Otsuka, 2008).

\subsection{The Need for the Visualization of Work Processes}

In actual corporate activity, there has been a growing trend towards the division of labor, and work is becoming increasingly dependent on the intuition and "knacks" possessed by multiple departments or people. In many cases, this kind of knowledge has become an implicit kind of knowledge. Also, different departments have different ways of doing things, so on a single project, there are no links in the work itself, and the actual work is being left entirely to the people performing it.

In order to understand the significance of the work and to steadily accomplish the existing work, it is important to make the work process more visible. By maintaining a constant awareness of the problems and carefully examining the work processes that have been made visible, companies will be able to achieve continuous improvements to the process, which will lead to the creation of new value.

Moreover, the visualization of the work process is also important for responding quickly to solve problems. If a company can create an environment in which anyone can see the state of progress of the work in a timely manner, thus speeding up the exposure of problems, and identify the context of the work at that point in time, it will be able to put effective countermeasures into place more quickly (Kanuma et al., 2006; Nakamura et al., 2008).

\section{CONSTRUCTING THE A-PPNS NAVIGATION SYSTEM TO REGENERATE WORK}

A navigation system for regeneration of software development work was built based on the A-PPNS conceptual model created from the analysis of the problems.

\subsection{Current Problems in Software Development}

This section will identify the challenges in the software development workplace, present a conceptual diagram of the problems currently faced by software development engineers and the solutions to those problems.

\subsubsection{Classifying Types of Work}

The work activities of companies today may be 
generally divided into the following ranks. Rank 1 tasks are simple tasks with no fluctuations in terms of cost and delivery timeframe, Rank 2 tasks are general tasks that do have fluctuations in terms of cost and delivery, and Rank 3 tasks are value-creation tasks with even greater differences in costs and delivery timeframes (Kato, 2007; Miwa, 2005).

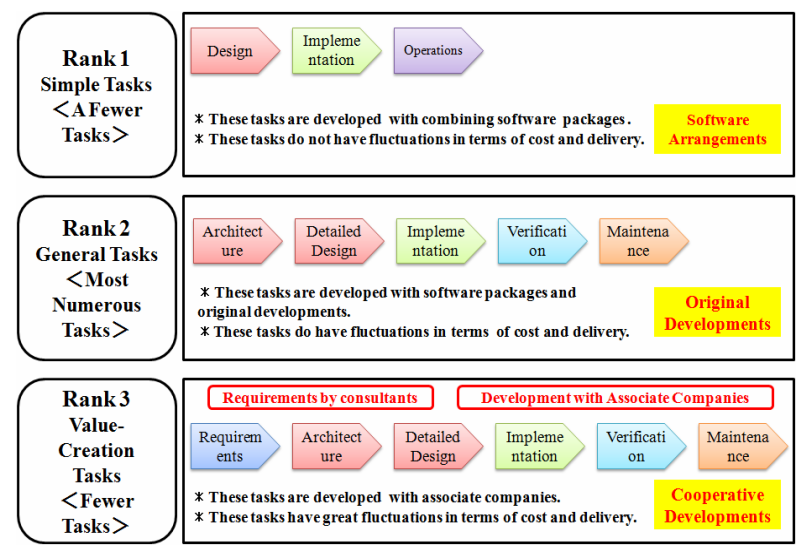

Figure 1. Ranking of Software Development Work.

In terms of work volume, there are fewer Rank 1 simple tasks and Rank 3 value-creation tasks; Rank 2 general tasks are by far the most numerous. Making improvements to Rank 2 general tasks every day and performing them efficiently form the essence of the regeneration of work within a company.

In recent times, the ability to perform Rank 3 value-creation tasks is in particular demand in the information services industry. For the revitalization of such work, understanding the significance of the Rank 2 tasks and accomplishing them properly is the most important challenge for achieving the creation of new value.

This article will deal with the Rank 2 general tasks shown in Figure 1. The authors have built a work process navigation system for the research industry, which lies at the core of business, as well as for development design for the manufacturing industry.

This article further takes this concept into the work of software development and presents a navigation system for such work (Kato, 2007; Nakamura et al., 2008).

\subsubsection{Using Workflow to Visualize Work}

We start by creating a workflow in order to visualize work processes and develop an effective strategy. Workflow visualization is a critical part of work innovation and reform, and is the basis for defining the requirements necessary to develop software (Kato, 2007; Nakamura et al., 2008; Otsuka, 2008). The workflow outlined in Figure 2 shows departments on the vertical

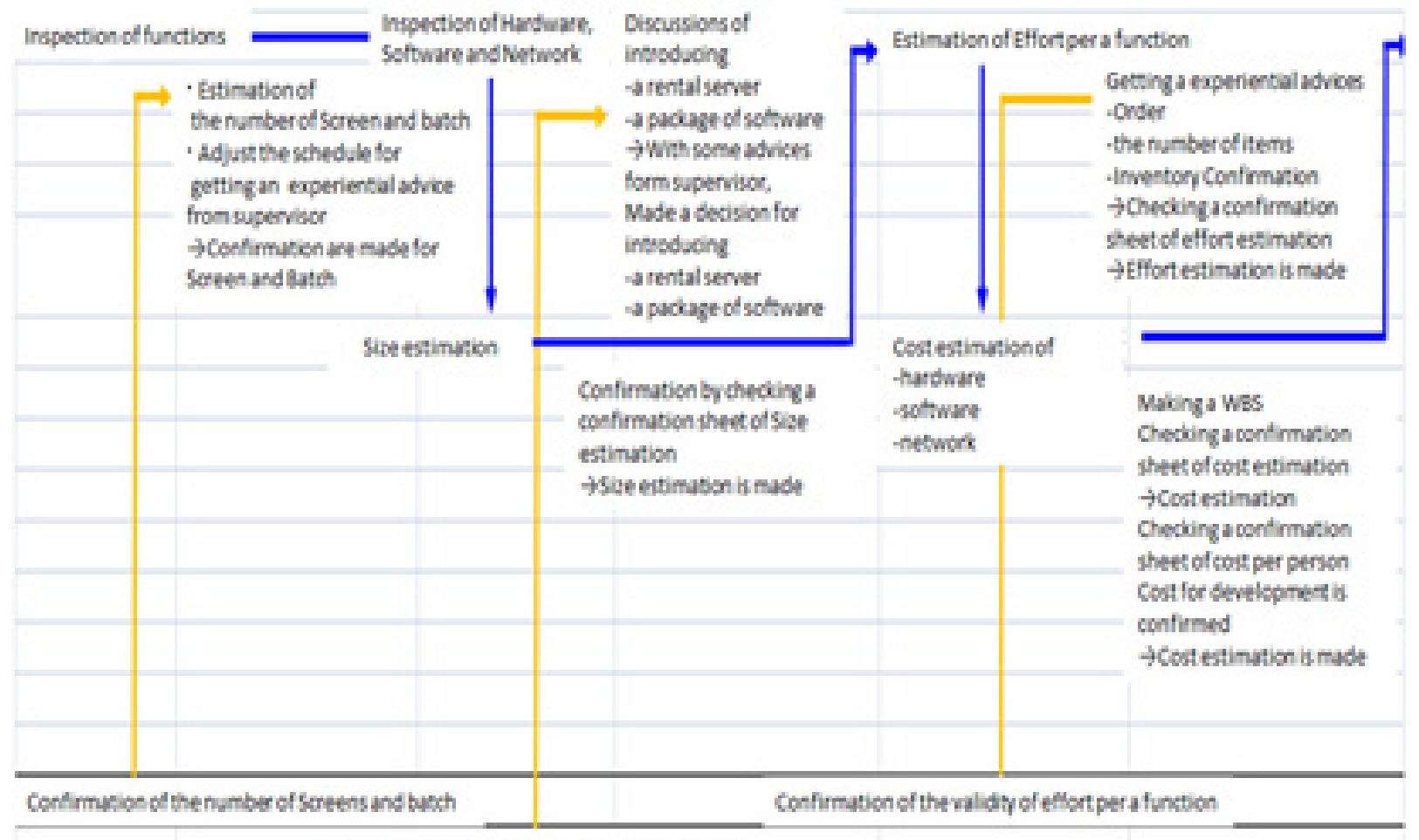

Suggestion for introduing a package soltware

Figure 2. Visualizing work using workflow. 


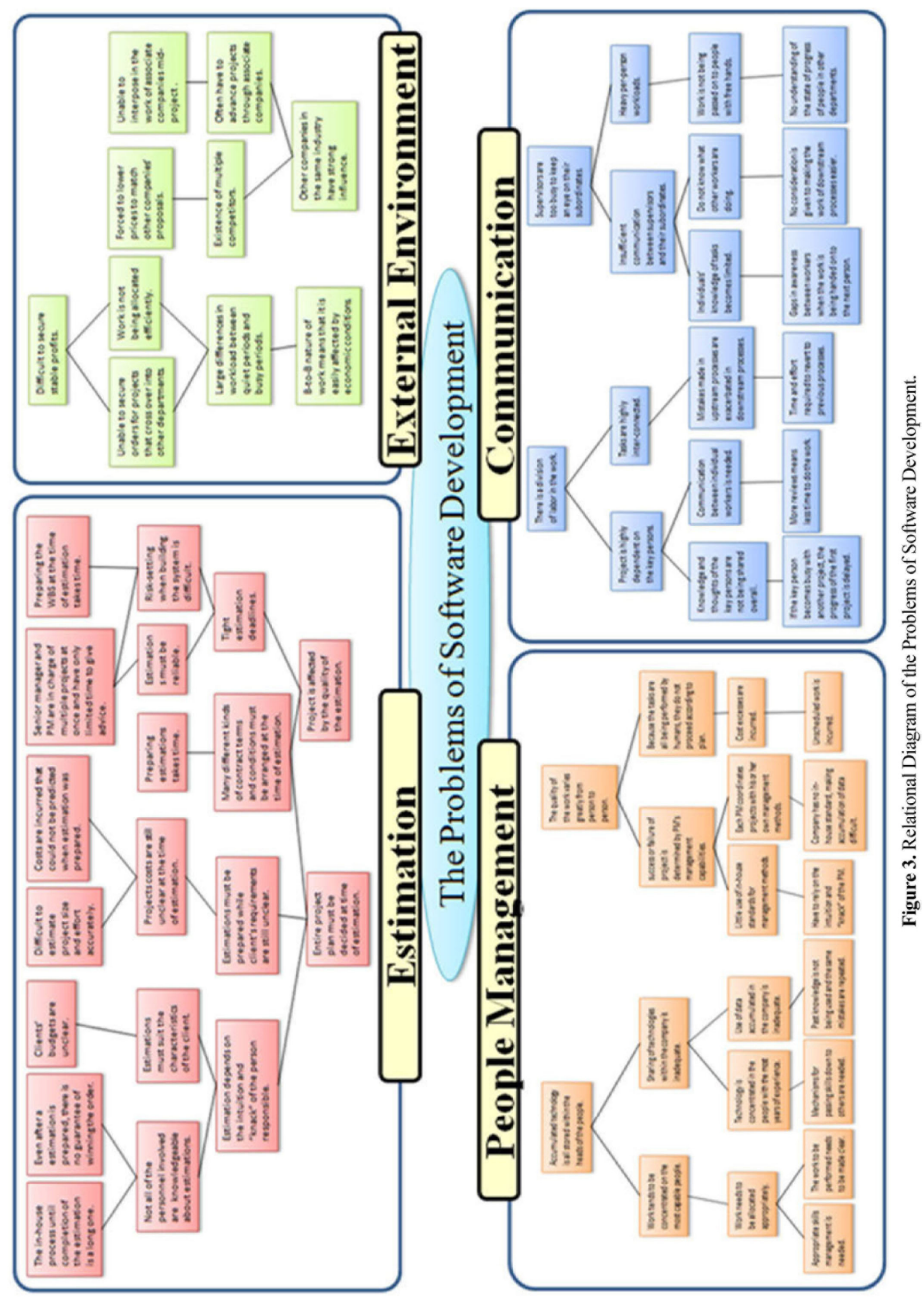




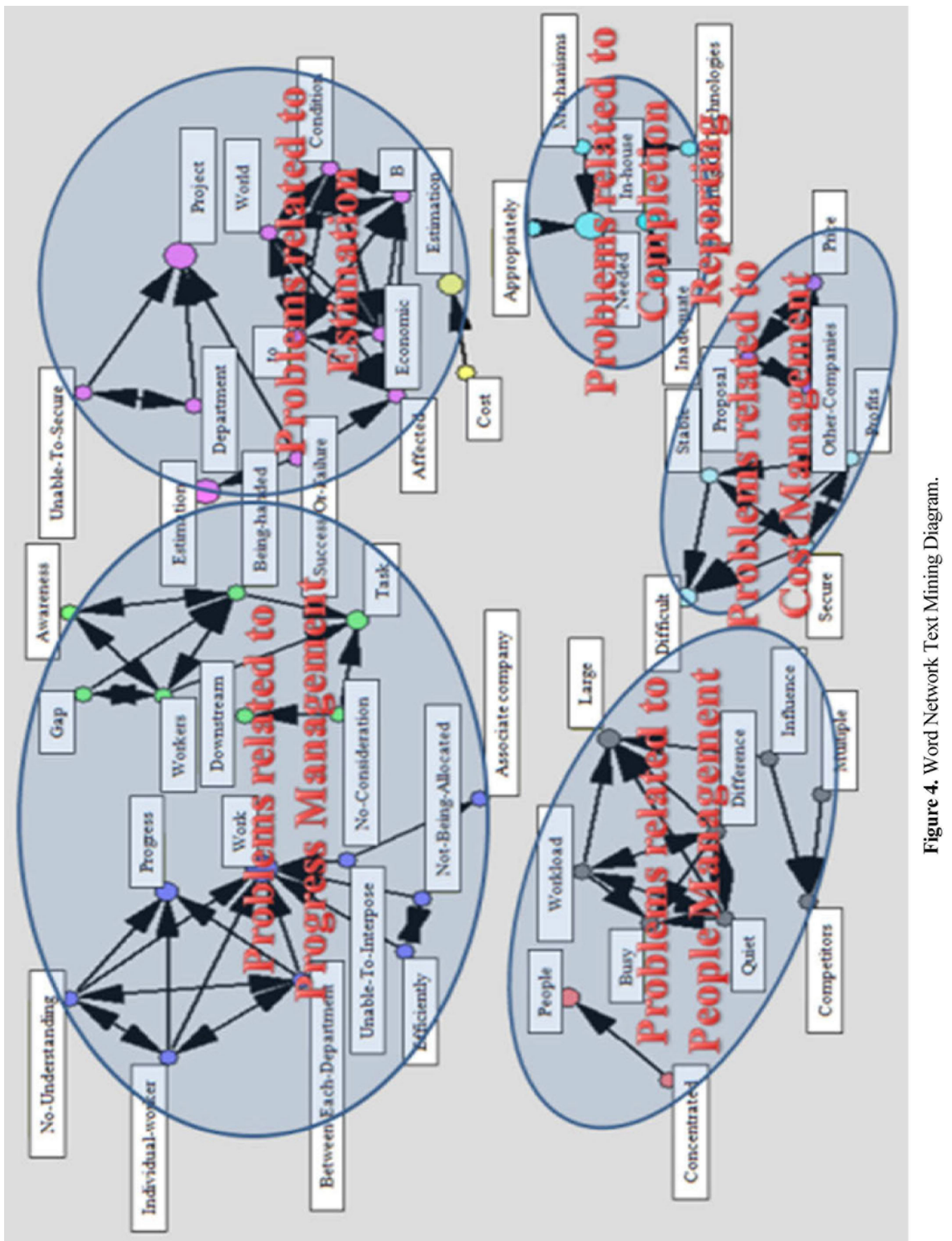


axis and time on the horizontal axis, allowing us to see the flow of information, objects, and people. The primary issue revealed by this workflow is the fact that the person in charge is checking with a skilled engineer to make sure that the number of screens is appropriate.

\subsubsection{Identifying Problems in Software Development}

To extract the problems in software development, a field survey was conducted. This study looked at twelve leading companies listed in the first and second section of the Tokyo Stock Exchange. The companies were all engaged in applying their unique technologies to specific customers and developing software to support their work processes. Face-to-face interviews of two hours in length were conducted with twelve skilled engineers from companies that are advanced in the field.

The problems raised by these engineers were compiled into a relational diagram. As shown in Figure 3, the problems were compiled onto 66 cards and broadly divided into four groups. Those four groups, along with the major cards they contain, are shown below.

In "Estimations," the main points are that not all of the workers involved are knowledgeable about estimations, the costs of the project are unclear at the time of estimating, and senior managers and PMs are in charge of multiple projects, so have only limited time to give advice.

In "People Management," the main problems cited are the need to clarify the work to be done, failure to make use of past knowledge resulting in the same mistakes being made, and a lack of standardized tools in the company making data accumulation difficult.

In "Communications," the interviewees cited the problems of progress in the project being delayed when the key persons become busy with other projects, increases in the number of reviews cutting back on the time available to do the work, and the inability to gauge the progress of work being done by people in other departments.

In "External Environment," the problems of the frequency of projects being pursued through partner companies and the inability to allocate work efficiently were cited. From the relational diagram, the challenges faced at the software development worksite were extracted. These were then analyzed using text mining methods.

\subsubsection{Analyzing Problems in Software Development}

The relational diagram in Figure 3 was analyzed with Word Network, the Text Mining Studio from Mathematical Systems, Inc. This resulted in the formation of nine clusters, as shown in Figure 4. These clusters were further divided into five groups. These groups are described below.

(1) Problems Related to Progress Management

Two clusters were combined to become problems related to progress management. In each of these two clusters, the arrows are concentrated on the words "pro- gress", "workers" and "work," which can be interpreted as problems with progress management, including the inability to obtain a grasp of how a project is progressing.

\section{(2) Problems Related to Estimations}

Two more clusters were combined into a group of problems related to estimations. With arrows concentrated on the words "estimation" and "project," these can be interpreted as problems related to estimations, such as the quality of an estimation affecting the project.

(3) Problems Related to Completion Reporting

In this cluster, the arrows are concentrated on the expressions, "in-house," "sharing of technologies," and "mechanisms," which can be interpreted as problems with completion reporting, such as that the mechanisms for the sharing of technologies is insufficient.

(4) Problems Related to Cost Management

Two clusters were combined to become problems related to cost management. Arrows were concentrated on the words "profits" and "price," leading to an interpretation of these problems as problems related to cost management, such as difficulties in securing stable profits.

(5) Problems Related to People Management

Two clusters were combined to become problems related to people management. Arrows were concentrated on the words "people," "workload," and "difference," leading to an interpretation of these problems as problems related to the management of people, such as large differences in workload between people.

\subsection{Organizing Problems in Software Development}

The problems revealed by the relational diagram and text mining analysis were organized into the structural diagram shown in Figure 5. The first layer contains the problems that emerged from the relational diagram, the second layer shows the types of related information, the third layer contains the extracted problems, and the fourth layer shows the solutions to those problems. Examining this structural diagram of the problems, it can be said that what software development work today needs are the unification of information, the construction of systems for sharing and data processing. From this structural diagram, a conceptual model for the APPNS to solve the problems in software development was built.

\subsection{Proposal of the A-PPNS Conceptual Model}

This section proposes the A-PPNS conceptual model and the A-PPNS Work Regeneration Approach.

\subsubsection{Outline of the A-PPNS}

Figure 6 shows the A-PPNS conceptual model for a navigation system for the regeneration of software development work. (i) Preparing a work process flow during planning will enable the current status of the work to 


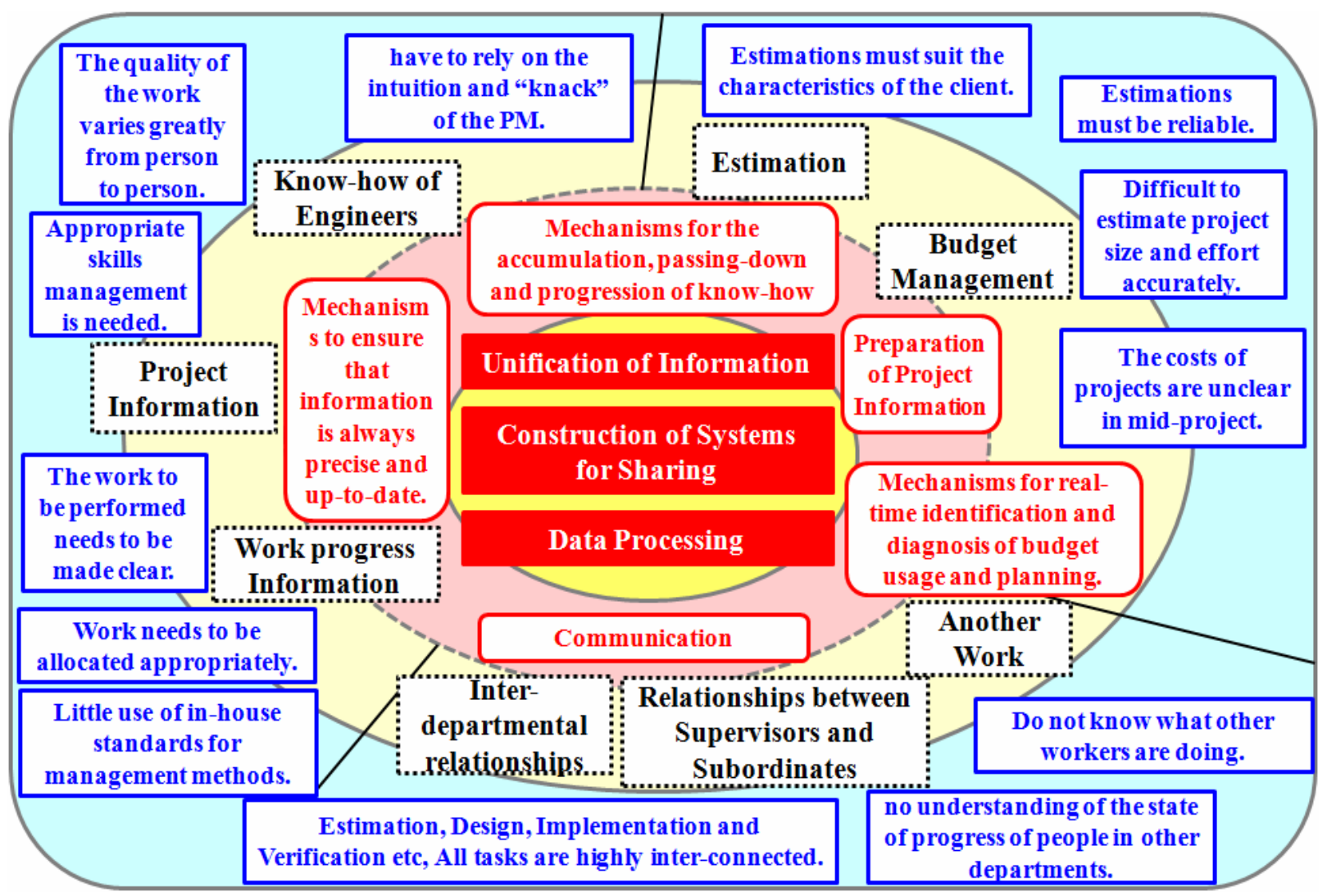

Figure 5. The Structural Diagram of the Problems in Software Development.

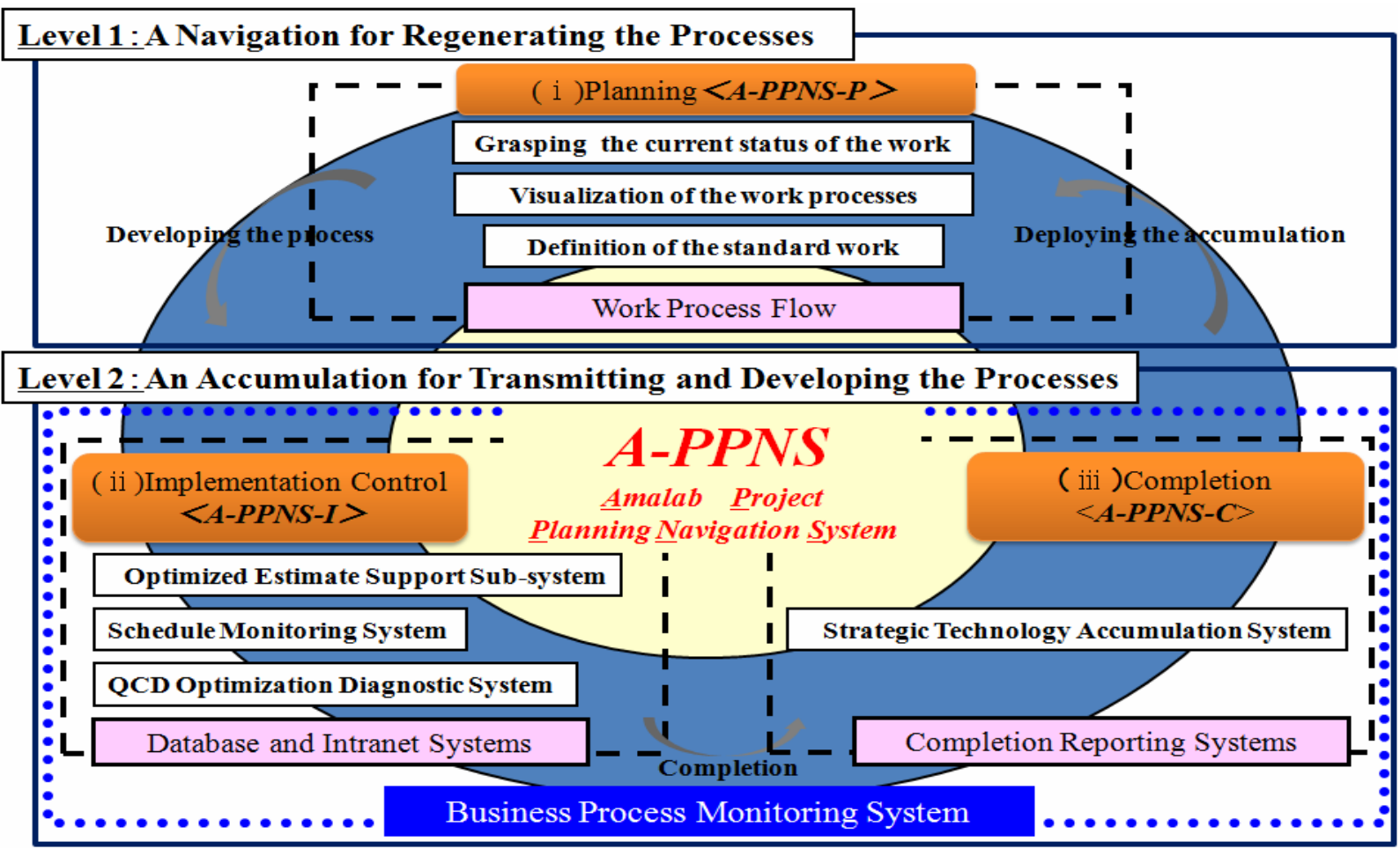

Figure 6. The A-PPNS Conceptual Model for a Work Regeneration Navigation System. 
be identified, define standard work and investigate current work processes. (ii) In Implementation Control, the A-PPNS-BPMS which is described later is used for the project information, to share across the entire company technological information such as the products (outcomes) of each work group, and what kind of characteristics each product has. Sharing process outcomes across the entire company will make it possible to assess not only the success or otherwise and the final results of the project but also the processes themselves. In budget management, spending of the budget can be projected along a timeline and the budget managed accordingly. (iii) At the conclusion of the project, the A-PPNSBPMS technology accumulation strategy application system is used to produce a report on the completion of the project. When the project ends, matters that presented technological problems in the project and the actions that were taken to respond to those problems are registered, making it possible to turn the implicit knowledge held by the people involved in the project into formal knowledge that can be used by the entire company.

Completion reporting is also simplified and the work made easier by examining the systems that were used during the Implementation Control phase. The technological information accumulated at this conclusion phase can then be used as the basis for strategies for winning orders for new projects. When estimations are being prepared for new project orders, the information registered in the technology accumulation strategy application system and the project information registered at the start of the project can be referred to and used. This will enable improvements in the efficiency and precision of estimation work (Amasaka, 2007; Miwa, 2005; Yoshikawa and Taura, 1997).

\subsubsection{The A-PPNS Work Regeneration Approach}

Figure 7 shows the A-PPNS software development work regeneration approach.

Step 1: is to produce an overall flowchart for the work. This will enable the visualization of the project based on personnel, materials, information and costs.

Step 2: the categorized work processes and the work information are deployed into the progress data base.

Step 3: the actual dates and times in which work processes were performed are input into the progress data base.

Step 4: when the work is completed, a completion report providing a summary of the work is input into the data base.

Step 5: the accumulated technology kept in the A-PPNSBPMS is used in new projects.

This describes the A-PPNS software development work regeneration approach, which assists work regeneration through the completion of Steps 1 through 5 (Kubota et al., 2005).

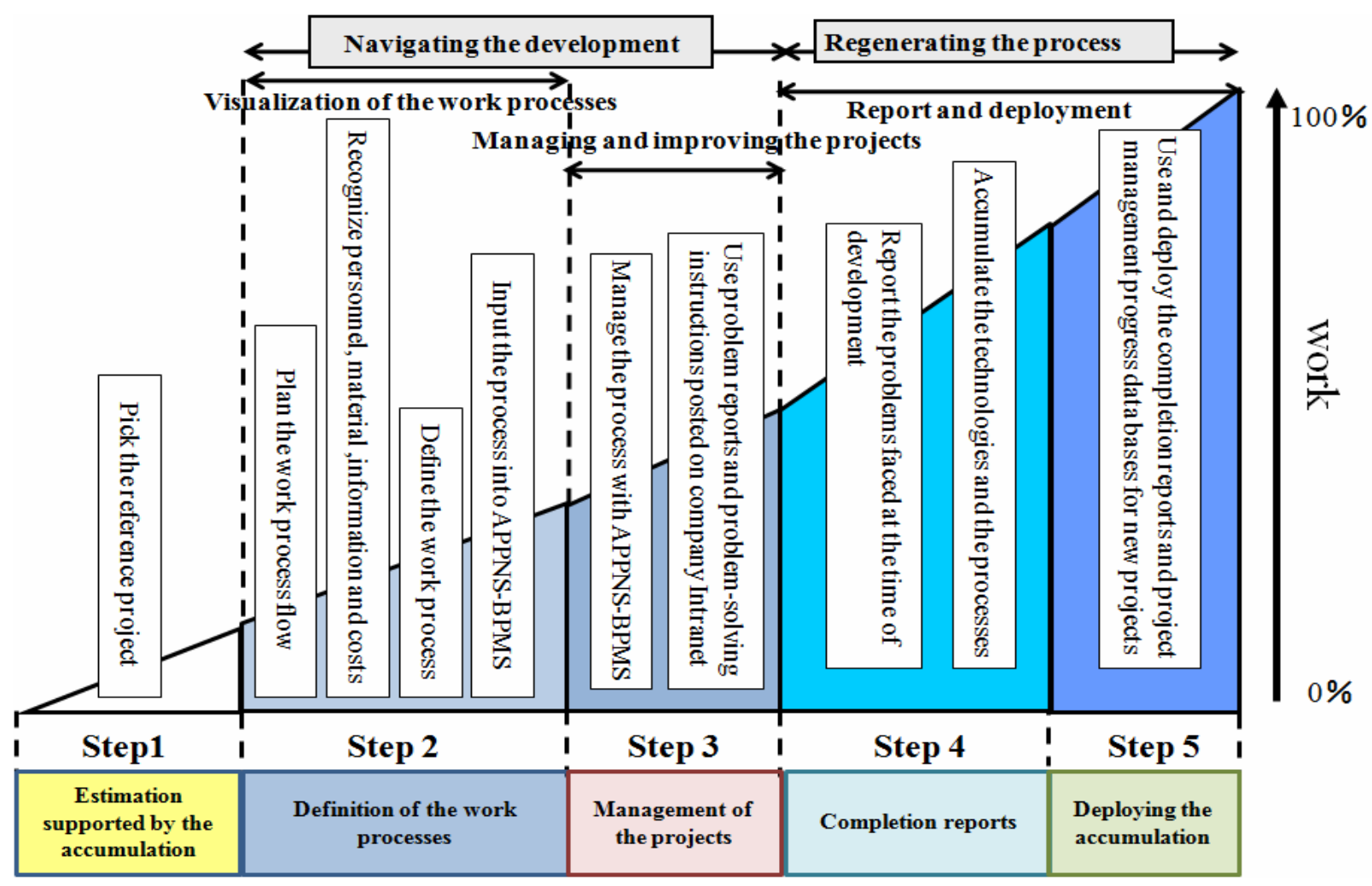

Figure 7. The A-PPNS Software Development Work Regeneration Approach. 


\section{APPLICATION}

The effectiveness of this system has been demonstrated and verified at Company A, a system integration company. In this application case study, the authors applied A-PPNS to actual work in each department in Company A. The effectiveness of A-PPNS was evaluated through verification interviews of the systems integrator. The authors have shown the effectiveness of the system through case studies applying the navigation systems.

\subsection{The A-PPNS-BPMS Business Process Monitoring System}

Figure 8 shows the A-PPNS-BPMS, which consists of the four cores of the A-PPNS navigation system for work regeneration. The four cores of this A-PPNS-BPMS each solve three problems.

(i) The Estimation Optimization Support SubSystem is used to take advantage of accumulated technology and knowledge and to improve estimation work efficiency and precision. Specifically, the sales representative who will present the estimation to the customer will use this system to refer to past projects and to the customer's characteristics, to diagnose whether the estimation is optimized for that customer and improve the precision of the estimation. The development department, which will prepare the detailed estimation, will use the technology accumulated from past projects to improve the efficiency of the estimation work.

(ii) The Schedule Monitoring System is used for the management of all projects that the company has received orders for, the management of progress of each project, and the management of the schedules of individual workers. Specifically, department general managers will use this system for strategies to secure new orders, section managers will use it for progress management of projects, and the individual workers will use it to check the current status of their own work.

(iii) The QCD Optimization Diagnostics System is used for quality achievement management, cost diagnosis and on-time completion support. Specifically, the development department will use this system to diagnose costs, and executives and managers will use it to obtain a grasp of an overall view, so that they can understand the QCD situation of all projects in progress at the company and issue instructions for improvement.

(iv) The Technology Accumulation Strategy System is used to accumulate technology from completed projects, use and manage engineer information and use and manage client information and characteristics strategies. Specifically, because all departments will have access to past projects, they will obtain technological information and work knowledge accumulated from past projects, which they can deploy and further develop in current projects.

Based on this conceptual model, the authors have built the A-PPNS-BPMS, a project management tool that embodies the systemized domains of the A-PPNS.

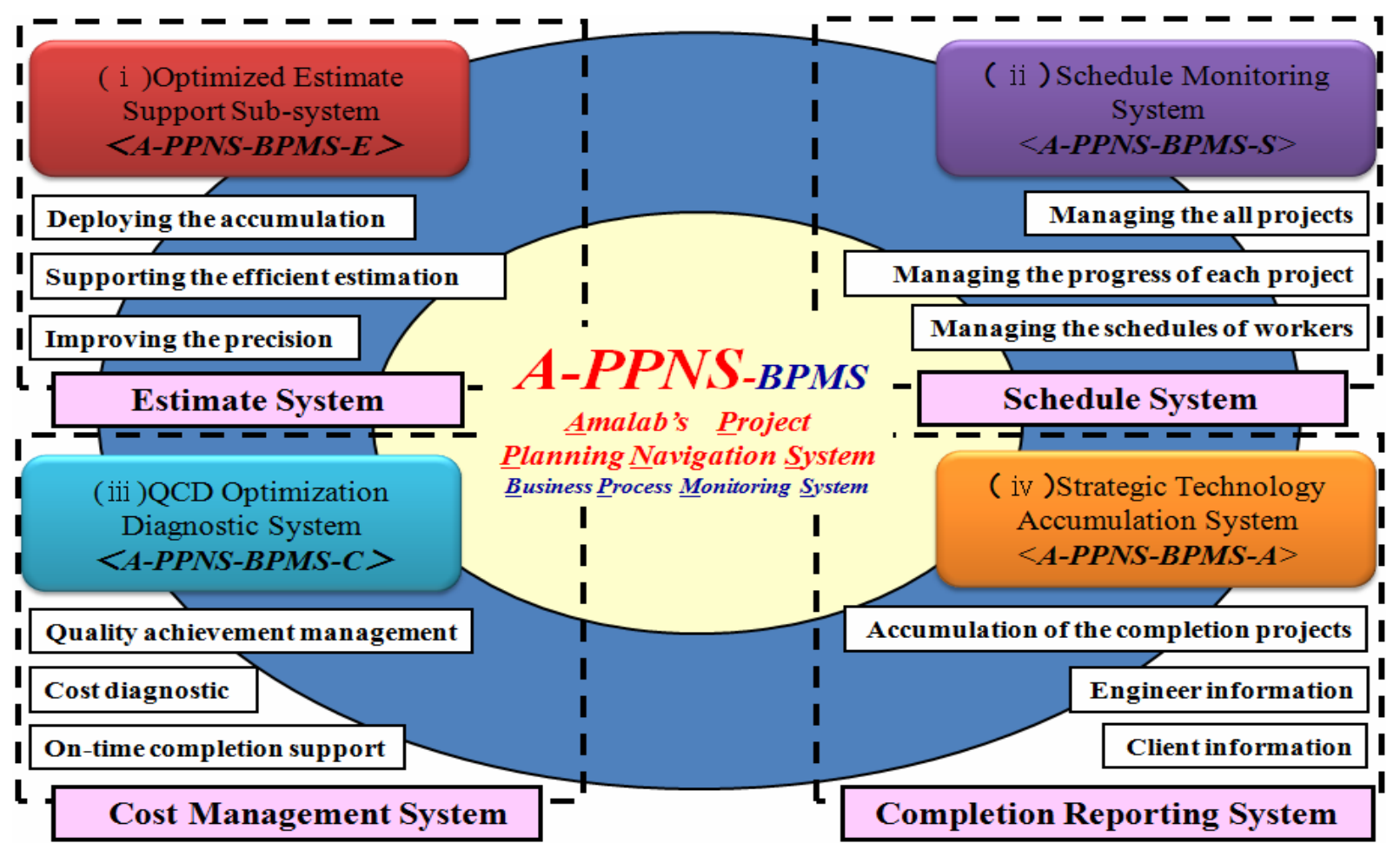

Figure 8. The Conceptual Model of the A-PPNS-BPMS Business Process Monitoring System. 


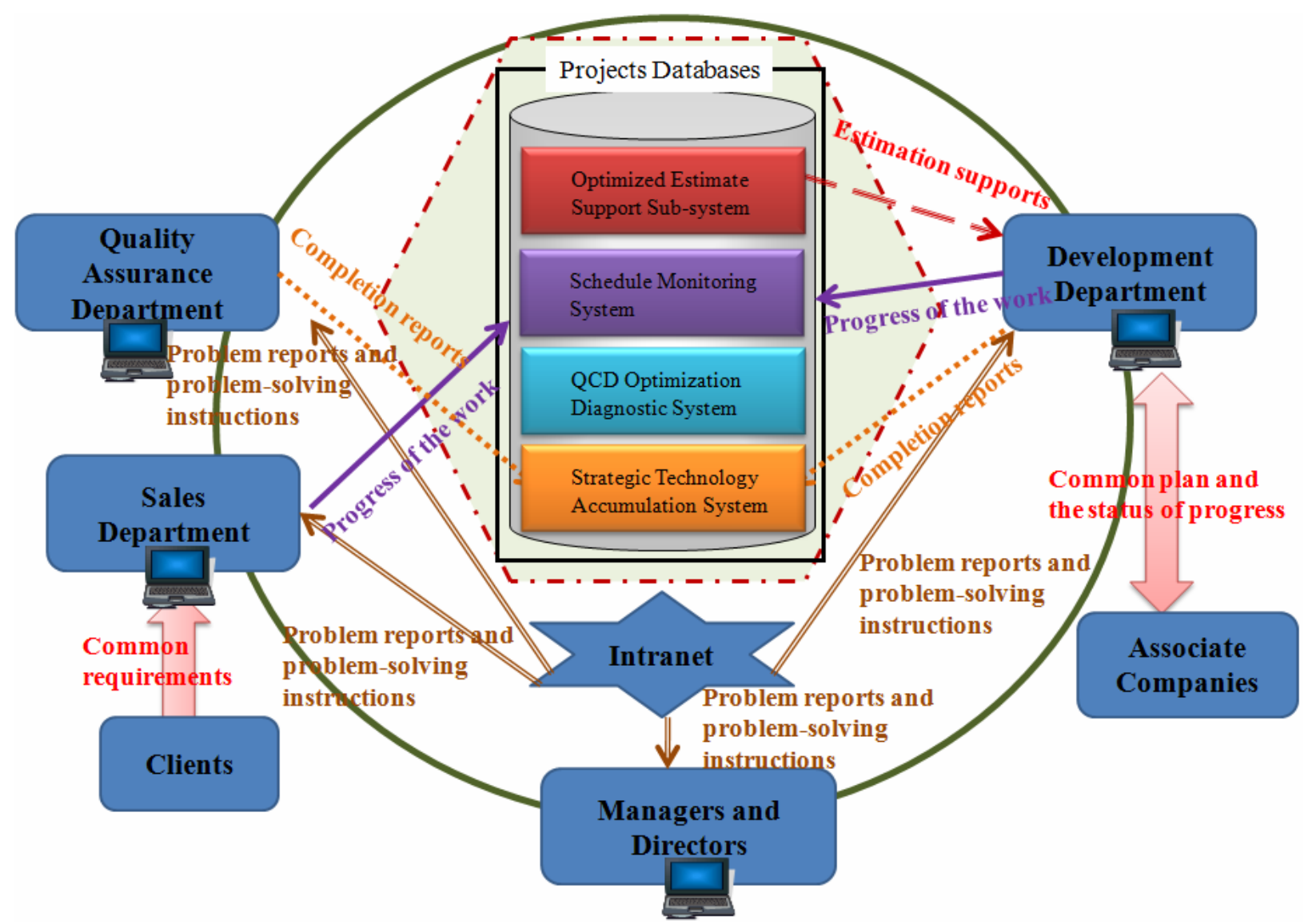

Figure 9. The A-PPNS Networking System.

\subsection{The A-PPNS Networking System}

Figure 9 shows the A-PPNS Networking System deployed to the actual work in Company A.

When a project commences, the sales department registers the requirement specifications of the project into the data base. Based on those specifications, each department produces a workflow, categorizes the work and sets standard times for each of the tasks based on actual measurement data and interviews.

What is important here is that the standard times should be set after first obtaining the agreement of the people who will perform that work. If aggressive standard times are set by calculating back from the delivery deadline, workers will be unable to keep to those standard times and the meaning of setting them in the first place will have been diluted.

Next, while the project is in progress, the actual time that the work has taken is also input. By displaying a comparison of the standard times and the actual times, the state of progress of the project can be monitored and managed. In this way, instead of each worker managing his or her progress in his or her own way, the state of progress of processes is visualized, and managers and executives are also able to obtain a grasp of the state of progress of the work in a timely manner. This will also shorten the time required for regular progress meetings.

Using a data base to manage the movements in and out of the office of personnel in the various departments (people flow) will make it possible to coordinate with other departments in a systematic manner. In particular, the schedule management of supervisors will become more important to the progress of the project, reducing the amount of time lost while waiting for approvals.

In budget management, the total of developer personnel costs and costs of goods purchased for each month are compiled into a graph and displayed for each date. This graph function uses three different graphs. One divides the project value into a daily amount to show the target figure, the second shows the actual total of personnel and goods purchased costs, and the third shows the projected figure that would result at the current rate of expenditure. These three graphs together give a visual representation of the costs.

After completion of the project, a completion report is created and input into the data base, thus completing the series of tasks. The information that has been input into the data base thus far is then viewable on the company's Intranet. Building this kind of network promotes the revitalization of communications within the company and assists managers and executives to manage the work processes of projects (Amasaka, 2005). 


\subsection{Verification Interviews with Systems Integrator}

Interviews were conducted of Company A, a systems integrator. The interviewees were skilled software development engineers. The verification was conducted by explaining the A-PPNS approach and the A-PPNSBPMS business process monitoring system built by the authors and conducting a face-to-face interview survey of their assessment of the systems' effectiveness in the software development workplace and any problems.

On a 4-point scale, the A-PPNS Software Development Work Regeneration Approach in Section 5 received the top score of 4 . The good points cited included that it appeared to be an efficient approach that would lead to regeneration of work in the software development workplace.

The APPNS-BPMS described in Section 4 received a score of 3 out of 4 . Challenges suggested by the interviewees included making the tool able to extract problems from current projects through analysis of the work.

\section{CONCLUSION}

In this article, the authors established A-PPNS, a navigation system for regenerating the software development business. The effectiveness of A-PPNS was verified at a specific systems integrator business and the given outcomes were obtained. The authors began with the visualization of the research industry and progressed to development design work and software development work. As an application case study, the navigation system built by the authors at Company B, a research company, was put into actual operation and the effectiveness of the system was confirmed. A development design work navigation system was also applied to a certain project in the manufacturing industry and its effectiveness was confirmed (Kato, 2007; Nakamura et al., 2008).

\section{REFERENCES}

Afsharian, S. and Giacomobono, M. (2008), A Framework for Software Project Estimation based on Cosmic, Dsm and Rework Characterization, Proceedings of the 1st international workshop on Business impact of process improvements, 15-23.

Amasaka, K. (2007), New japan model- "Science TQM": Theory and practice for strategic quality management, Study group of the ideal situation: the quality management of the manufacturing industry, Maruzen, 207-224 (in Japanese).

Amasaka, K. (2005), Constructing a Customer Science Application System "CS-CIANS"-Development of a Global Strategic Vehicle "Lexus" Utilizing New JIT-, WSEAS Transactions on Business and Eco- nomics, 2, 135-142.

Changa, C. K., Jianga, H., Dib, Y., Zhuc, D., and Ged, Y. (2008), Time-line Based Model for Software Project Scheduling with Genetic Algorithms, Information and Software Technology, 50, 1142-1154.

Galorath, D. D. and Evans, M. W. (2008), Software Sizing, Estimation, and Risk Management, Auerbach Publications.

Heričko, M. and Živkoviča, A. (2008), The Size and Effort Estimates in Iterative Development, Information and Software Technology, 50, 772-781.

Heričko, M., Živkoviča, A., and Rozmana, I. (2008), An Approach to Optimizing Software Development Team Size, Information Processing Letters, 108, 101-106.

Kato, K. (2007), Study of a Process Visualization for Research Business, A Thesis for Master's Degree Amasaka-laboratory of Aoyama Gakuin University (in Japanese).

Kanuma, Y., Suzuki, Y., Ishizuka, N., and Kato, T. (2006), Establishment of a Visualization System of Quality Information, Proceedings of The 36th Annual Conference of the Japanese Society for Quality Control.

Kubota, A., Okada, K., Sekiguchi, K., and Imanoya, F. (2005), A Case of Business Process Innovation for Product Development in Semiconductor and Integrated Circuit Business, Journal of the Society of Project Management, 7, 38-43.

Mittas, N. and Angelis, L. (2010), Visual Comparison of Software Cost Estimation Models by Regression Error Characteristic Analysis, Journal of Systems and Software, 83, 621-637.

Miwa, S. (2005), Study of Appraisal Standard Model, Considering the Quality and the Price, A Thesis for Master's Degree Amasaka-laboratory of Aoyama Gakuin University(in Japanese).

Nakamura, M., Yamaji, M., and Amasaka, K. (2008), Study of Approach Method for a Navigation System in Development Design Business, Proceedings of The 38th Annual Conference of the Japanese Society for Quality Control, 161-164(in Japanese).

Otsuka, H. (2008), System Development Methodology making Effective Use of Intellectual Property, Unisys Technology Review, Nihon Unisys Ltd., 28, 314(in Japanese).

Pendharkar, P. and Rodger, J. (2009), The Relationship between Software Development Team Size and Software Development Cost, Communications of the ACM-Rural engineering development, 52, 141144.

Yoshikawa, H. and Taura, T. (1997), New Engineering Knowledge-Essence of Technology Knowledge, A Nature of the Context and the Creativity-, Publish Association of University of Tokyo, 161-188(in Japanese). 